\title{
Suppressed Neuronal Activity and Concurrent Arteriolar Vasoconstriction May Explain Negative Blood Oxygenation Level-Dependent Signal
}

\author{
Anna Devor, ${ }^{1,4}$ Peifang Tian, ${ }^{1}$ Nozomi Nishimura, ${ }^{2}$ Ivan C. Teng, ${ }^{1}$ Elizabeth M. C. Hillman, ${ }^{4}$ S. N. Narayanan, ${ }^{4}$ \\ Istvan Ulbert, ${ }^{4,5}$ David A. Boas, ${ }^{4}$ David Kleinfeld, ${ }^{2}$ and Anders M. Dale ${ }^{1,3}$ \\ Departments of ${ }^{1}$ Neurosciences, ${ }^{2}$ Physics, and ${ }^{3}$ Radiology, University of California, San Diego, California 92093, ${ }^{4}$ Massachusetts General Hospital, Harvard \\ Medical School, Charlestown, Massachusetts 02129, and 5 Institute for Psychology of the Hungarian Academy of Sciences, Budapest 1068, Hungary
}

\begin{abstract}
Synaptic transmission initiates a cascade of signal transduction events that couple neuronal activity to local changes in blood flow and oxygenation. Although a number of vasoactive molecules and specific cell types have been implicated, the transformation of stimulusinduced activation of neuronal circuits to hemodynamic changes is still unclear. We use somatosensory stimulation and a suite of in vivo imaging tools to study neurovascular coupling in rat primary somatosensory cortex. Our stimulus evoked a central region of net neuronal depolarization surrounded by net hyperpolarization. Hemodynamic measurements revealed that predominant depolarization corresponded to an increase in oxygenation, whereas predominant hyperpolarization corresponded to a decrease in oxygenation. On the microscopic level of single surface arterioles, the response was composed of a combination of dilatory and constrictive phases. Critically, the relative strength of vasoconstriction covaried with the relative strength of oxygenation decrease and neuronal hyperpolarization. These results suggest that a neuronal inhibition and concurrent arteriolar vasoconstriction correspond to a decrease in blood oxygenation, which would be consistent with a negative blood oxygenation level-dependent functional magnetic resonance imaging signal.
\end{abstract}

Key words: hemodynamic; somatosensory; two-photon; voltage-sensitive dyes; intrinsic imaging; electrode array

\section{Introduction}

Changes in neuronal activity in the cerebral cortex are coupled to changes in the blood flow and oxygenation (Roy and Sherrington, 1890). It is generally accepted that synaptic release associated with neuronal communication initiates a chain of events that transmit the signal to nearby blood vessels. Previous studies have demonstrated changes in diameter and velocity of blood flow in single arterioles evoked by neuronal, glial, and pharmacological stimuli in vitro (Simard et al., 2003; Zonta et al., 2003; Cauli et al., 2004; Mulligan and MacVicar, 2004; Filosa et al., 2006; Rancillac et al., 2006) and in vivo (Faraci and Breese, 1993; Kleinfeld et al., 1998; Chaigneau et al., 2003; Takano et al., 2006). Recently, the range of blood vessels capable of flow control has been extended to include capillaries (Peppiatt et al., 2006). Potential molecular players in the neurovascular signal transduction pathway(s) have also been implicated (for review, see Iadecola, 2004; Lauritzen, 2005; Hamel, 2006). Nevertheless, in vivo transformation of stimulus-induced activation of neuronal cir-

Received Jan. 11, 2007; revised March 17, 2007; accepted March 19, 2007.

This work was supported by the National Institute of Neurological Disorders and Stroke (NS-051188 to A.D., NS053684 to E.H.), National Institute of Biomedical Imaging and Bioengineering (EB00790 to A.M.D., EB003832 to D.K.), and National Center for Research Resources (RR021907 to D.K.). We thank Earl Dolnick for excellent technical support of two-photon microscopy instrumentation.

Correspondence should be addressed to either of the following: Anna Devor or David Kleinfeld, University of California at San Diego, 9500 Gilman Drive, La Jolla, CA 92093; E-mail: adevor@ucsd.edu or dk@physics.ucsd.edu. D0I:10.1523/JNEUROSCI.0134-07.2007

Copyright $\odot 2007$ Society for Neuroscience $\quad$ 0270-6474/07/274452-08\$15.00/0 cuits into macroscopic hemodynamic changes such as those observed using blood oxygenation level-dependent (BOLD) functional magnetic resonance imaging (fMRI) is still unclear. For example, it is not known whether activation seen in fMRI images should be interpreted as excitation or inhibition at the level of interactions between brain neurons (for review, see Raichle and Mintun, 2006).

A number of previous studies have demonstrated that a somatosensory stimulus perturbs an intricate balance of excitation and inhibition in neocortex eliciting a mixture of excitatory and inhibitory postsynaptic potentials in primary somatosensory cortex (SI) (Brecht and Sakmann, 2002; Brecht et al., 2003; Manns et al., 2004). The excitation and inhibition are present both in principal cortical columns, receiving direct thalamic input, and in the surround columns, receiving the input through lateral corticocortical connections (Moore and Nelson, 1998). The ratio of excitation to inhibition decreases as a function of distance from the center, creating the "surround" inhibition (Simons and Carvell, 1989; Kleinfeld and Delaney, 1996; Takashima et al., 2001; Derdikman et al., 2003).

Previously, using intrinsic optical imaging, Devor et al. (2005) demonstrated an antagonistic center-surround spatial pattern of the hemodynamic response to a localized somatosensory stimulus. Stimulus-evoked decrease in blood oxygenation had been also observed by other groups using fMRI (Harel et al., 2002; Shmuel et al., 2002, 2006). In the present study we demonstrate that the surround stimulus-induced functional decrease in blood oxygenation and volume corresponds to a neuronal inhibition 
and arteriolar vasoconstriction. Because arterioles have a wall of smooth muscle and their diameter is controlled by vasoactive substances (Faraci and Heistad, 1998; Hamel, 2006), this vasoconstriction is likely to reflect an activation of signaling pathways presumably initiated by neuronal release of neurotransmitters and neuropeptides associated with functional inhibition (Faraci and Breese, 1993; Cauli et al., 2004). Moreover, because synaptic activity is associated with increased metabolism (Attwell and Iadecola, 2002), and because no decrease in spiking rates was observed, our results also suggest that the milieu of signaling molecules released during synaptic transmission, rather than energy consumption feedback, controls the hemodynamic response.

\section{Materials and Methods}

Animal preparation. Sprague Dawley rats ( $n=15 ; 230-310 \mathrm{~g})$ were used. Among them, four were used for the spectroscopic imaging, four in voltage-sensitive dye (VSD) experiments, and seven for two-photon microscopy. All experimental procedures were approved by the Massachusetts General Hospital Subcommittee on Research Animal Care and the University of California at San Diego Institutional Animal Care and Use Committee. Rats were initially anesthetized with halothane, $1.5 \%(\mathrm{v} / \mathrm{v})$ initially and $1 \%$ during ventilation, or isofluorane, $3 \%(\mathrm{v} / \mathrm{v})$ initially and $2 \%$ during ventilation, and ventilated with mixture of air and oxygen during surgical procedures. During the surgery, cannulas were inserted in the femoral artery and vein. Halothane or isofluorane was discontinued, and anesthesia was maintained with $50 \mathrm{mg} / \mathrm{kg}$ intravenous bolus of $\alpha$-chloralose followed by continuous intravenous infusion at 40 $\mathrm{mg} \cdot \mathrm{kg}^{-1} \cdot \mathrm{h}^{-1}$. Heart rate, blood pressure, and body temperature were continuously monitored. In VSD and spectroscopic imaging experiments, blood gas was analyzed. Respiration was aimed to maintain $\mathrm{Pa}_{\mathrm{CO}_{2}}$ between 35 and $45 \mathrm{mmHg}, \mathrm{Pa}_{\mathrm{O}_{2}}$ between 140 and $180 \mathrm{mmHg}$, and $\mathrm{pH}$ between 7.35 and 7.45 .

In experiments involving spectroscopic imaging, an area of skull overlying the primary somatosensory cortex was exposed and then thinned until soft and transparent. A well of dental acrylic was built and filled with mineral oil (Sigma, St. Louis, MO). In experiments involving VSD and two-photon microscopy, the thinned skull and dura matter were removed. To avoid herniation of the exposed brain caused by excessive intracranial pressure, CSF was drained from the fourth cerebral ventricle.

We chose forepaw stimulus to increase the signal-to-noise ratio (SNR) of our measurements. The stimulus consisted of a train of three electrical pulses ( $3 \mathrm{~Hz}, 300 \mu \mathrm{s}, 0.5-1.5 \mathrm{~mA}$ ) delivered to a forepaw through a pair of thin needles implanted under the skin. The intensity was adjusted to provide stimulation below the movement threshold. The interstimulus interval (ISI) was kept at $20 \mathrm{~s}$ during imaging of hemodynamic signals. Electrophysiological trials acquired at $20 \mathrm{~s}$ ISI did not reveal quantitative difference to those acquired at 5 or $1 \mathrm{~s}$ ISI. Therefore, VSD imaging and laminar electrophysiology was acquired using 5 and $1 \mathrm{~s}$ ISI, respectively, to optimize for number of trials.

Spectroscopic optical imaging. Spectroscopic imaging is described in detail by Dunn et al. (2003). Briefly, light from a tungsten-halogen lamp was directed through a six-position rotating filter wheel $(560,570,580$, $590,600$, and $610 \mathrm{~nm})$ coupled to a $12 \mathrm{~mm}$ fiber bundle. Images of $\sim 6 \times$ $6 \mathrm{~mm}$ area $(\sim 150 \times 150$ pixels $)$ containing the thin-skull exposure were acquired by cooled 12-bit CCD camera (Coolsnap; Photometrics, Tucson, AZ). Image acquisitions were triggered at $\sim 15 \mathrm{~Hz}$ by individual filters in the filter wheel passing through an optic sensor. The spectral data were converted to percent-change maps for oxyhemoglobin $(\mathrm{HbO})$, deoxyhemoglobin $(\mathrm{Hb})$, and total hemoglobin ( $\mathrm{HbT}$ ) using the modified Beer-Lambert law. Differential path-length correction was applied to adjust for the differential optical path length through the tissue at different wavelengths. Dense mapping using extracellular microelectrodes (2-4 M $\Omega$; FHC, Bowdoinham, ME) was performed at the end of each spectroscopic imaging session to determine the location of maximal neuronal response (Devor et al., 2005).

Voltage-sensitive dye imaging. The well was filled with artificial CSF (ACSF) (Kleinfeld and Delaney, 1996) immediately after exposure. Commercially available "blue" voltage-sensitive dye RH-1691 (Optical Imag- ing, Mountainside, NJ) was dissolved in ACSF, and the well was filled with $100-200 \mu \mathrm{l}$ of the dye solution. The dye was left for $1.5-2 \mathrm{~h}$ to impregnate the brain. The staining solution in the well was then replaced a number of times with fresh ACSF to remove any unbound dye molecules.

Images of $\sim 6 \times 6 \mathrm{~mm}$ area $(\sim 100 \times 100$ pixels $)$ containing the exposure were acquired using a commercial CCD-based VSD imaging system (Optical Imaging, New York, NY). Illumination light from a tungstenhalogen lamp was passed through an excitation filter $(630 \mathrm{~nm})$. The detected signal was passed through a filter cube consisting of a dichroic mirror and an emission filter $(665 \mathrm{~nm})$. Data acquisition was triggered on respiration and heart beat. Stimulus trials were interleaved with blank (no-stimulus) trials. Each condition (stimulus and blank) was divided by the prestimulus baseline. Then, blanks were subtracted from the stimulus to eliminate respiration and heart beat artifacts. Dense mapping using extracellular microelectrodes (2-4 M $\Omega$; FHC) was performed at the end of each VSD imaging session to confirm the location of maximal neuronal response.

Electrode array recordings. Electrophysiological recordings were performed in two animals after VSD imaging using two linear array multielectrodes with 23 contacts spaced at $100 \mu \mathrm{m}$ (Ulbert et al., 2001). The first array was inserted at the location of the maximal neuronal response as identified using previous mapping with an extracellular microelectrode (2-4 M $\Omega$; FHC). The second array was inserted $\sim 3 \mathrm{~mm}$ posterior relative to the first within the region of surround hyperpolarization. The depth was estimated based on a contact number when contact 1 was positioned at the cortical surface using visual control. The recorded potential was amplified and filtered into two signals: a low-frequency part $(0.1-500 \mathrm{~Hz}$, sampled at $2 \mathrm{kHz}$ with 16 bits) and a high-frequency part (150-5000 Hz, sampled at $20 \mathrm{kHz}$ with 12 bits) (for details, see Ulbert et al., 2001). The low-frequency part is referred to as the local field potential (LFP). The high-frequency part was further filtered digitally between 750 and $5000 \mathrm{~Hz}$ using a zero phase-shift second-order Butterworth filter, and then rectified along the time axis to provide the multiple unit activity (MUA). Finally, the MUA data were smoothed along the time axis using a Gaussian kernel of $1 \mathrm{~ms}$ full width at $e^{-1}$ of peak amplitude.

Two-photon microscopy. A chamber consisting of a metal frame (Kleinfeld and Delaney, 1996) and a removable glass coverslip (number 1) lid was glued to the skull. The space between the exposed brain surface and the coverglass was filled with $0.7 \%(\mathrm{w} / \mathrm{v})$ agarose (Sigma) in ACSF. To visualize the vasculature, $\sim 0.3 \mathrm{ml}$ of $5 \%$ (w/v) solution of $2 \mathrm{MDa}$ fluorescein-conjugated dextran (FD-2000S; Sigma) in physiological saline was injected intravenously (Nishimura et al., 2006). The dye labeled blood plasma, whereas blood cells looked like dark shadows on the bright background.

Images were obtained using home built two-photon laser-scanning microscope (Tsai et al., 2002) as described previously (Schaffer et al., 2006). We used a $4 \times$ air objective [XLFluor $4 \times / 340$; numerical aperture (NA), 0.28; Olympus, Tokyo, Japan] to obtain images of the surface vasculature across the entire cranial window to aid in navigating around the cortical vasculature. Ten-times (Achroplan; NA, 0.3; Zeiss, Oberkochen, Germany) and $20 \times$ (NA, 0.5; Olympus) water-immersion objectives were used for high-resolution imaging and line scan measurements. For diameter measurements $<500 \mu \mathrm{m}$ long, line scans were acquired across multiple vessels (up to six) with a scan rate of $1.3 \mathrm{kHz}$. The scan resolution was $0.6 \mu \mathrm{m}$ or less. One to two locations (arterioles) per animal were measured at $\sim 1 \mathrm{~h}$ intervals; the data acquisition was terminated when the response amplitude at these "control" locations started to decrease indicating a general deterioration of physiological conditions. Data analysis was performed in Matlab environment.

Surface potentials recordings. Recordings were done using a silver ball electrode. Ball electrode recordings were performed immediately before two-photon microscopy from 10 to 15 locations within an $\sim 6 \times 6 \mathrm{~mm}$ exposure within the recording/imaging chamber. The electrode was positioned in direct contact with the cortical surface. The surface was kept moist with repeated applications of ACSF. The recording locations were registered relative to the cortical microvasculature for comparison with two-photon microscopy data. The signals were amplified and filtered between $0.1 \mathrm{~Hz}$ and $10 \mathrm{kHz}$. 


\section{Results \\ Spectroscopic imaging of \\ blood oxygenation}

We imaged oxyhemoglobin ( $\mathrm{HbO})$, deoxyhemoglobin $(\mathrm{Hb})$, and total hemoglobin ( $\mathrm{HbT}$ ) changes in response to forepaw somatosensory stimulus. All three spectroscopic measures ( $\mathrm{HbO}, \mathrm{Hb}$, and $\mathrm{HbT}$ ) showed an antagonistic center-surround activation pattern in which the central area of the response was surrounded by an area of the opposite sign (Fig. 1A). For example, the area of increased oxygenation (Fig. $1 A$, red) was surrounded by an area of decreased oxygenation (blue). The imaging session was followed by mapping the center of the evoked neuronal activity using extracellular electrodes (see Materials and Methods). The center "positive" response always overlapped with contralateral forepaw neuronal representation (data not shown).

Response time courses were extracted from $0.5 \mathrm{~mm}$ rings around the center of the hemodynamic response within the exposure. Specifically, we collapsed the spatial dimension by averaging all pixels within a ring (Fig. $1 B$ ). The center was estimated as the peak of the earliest detectable $\mathrm{HbT}$, because HbT had the highest SNR compared with $\mathrm{HbO}$ and $\mathrm{Hb}$. The rings are superimposed on images in Figure $1 A$. The surround response had a smaller amplitude and a diffuse pattern. Peak positive and peak negative signals were measured within each ring. The results for $\mathrm{HbO}$ from four animals are superimposed in Figure $1 C$ (see also supplemental Fig. 1, available at www. jneurosci.org as supplemental material). The positive signals (evoked oxygenation increase) declined gradually as a function of distance (ring number). However, negative signals (evoked oxygenation decrease) were observed at distances $>2 \mathrm{~mm}$.

\section{Voltage-sensitive dye imaging}

To investigate the neuronal correlate of the center positive and the surround negative hemodynamic response we used VSD imaging that measured mostly subthreshold membrane polarization in upper cortical layers (Ferezou et al., 2006).

The measurements were performed under the same conditions as the hemodynamic imaging in a separate group of animals. The response to each stimulus in a train of three stimuli was composed of the initial depolarization followed by a hyperpolarization (Fig. 2). The earliest detectable response was spatially localized (Fig. 2A) $(t=10 \mathrm{~ms})$ and overlapped with contralateral forepaw neuronal representation (data not shown). The subsequent spatial spread of the signal (Fig. $2 A$ ) can be explained by a substantial horizontal spread of the apical dendrites of layer II/III pyramidal neurons (Petersen et al., 2003) and extensive corticocortical lateral connections (Brecht et al., 2003; Manns et al., 2004). The subsequent hyperpolarization is indicative of neuro- nal inhibition ( $t>100 \mathrm{~ms}$ ) (Grinvald et al., 1982). The strongest hyperpolarization was observed in a region surrounding the center of neuronal response.

To quantify the depolarization and hyperpolarization as a function of distance from the center, we extracted time courses from $0.5 \mathrm{~mm}$ concentric rings around the center of the response within the exposure (Fig. 2 B, as in the case of the hemodynamic measures in Fig. 1). We used a somewhat smaller exposure size in VSD experiments because of surgical limitations related to full exposure (removal of the bone and dura matter). The center was estimated as the peak of the first detectable depolarization (at $t=$ $10 \mathrm{~ms}$ ). Normalization of the initial depolarization amplitude revealed that, relative to the initial depolarization, the subsequent hyperpolarization was greater in the surround regions (Fig. $2 \mathrm{~B}$, right, black arrows). Peak depolarization and peak hyperpolarization were measured within each ring. The results from four subjects are superimposed in Figure 2C. The peak depolarization declined gradually as a function of distance. However, the strongest surround hyperpolarization tended to occur at $2.0-3.5 \mathrm{~mm}$ (Fig. 2C, top). The ratio of peak hyperpolarization to peak depo- 
A

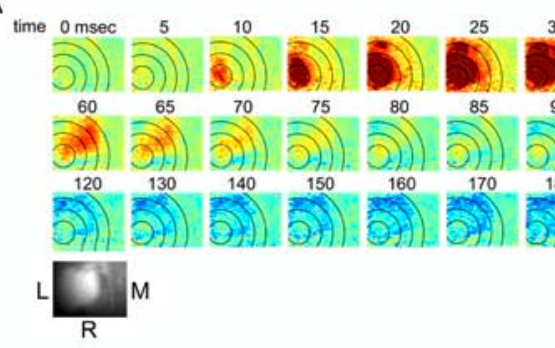

B

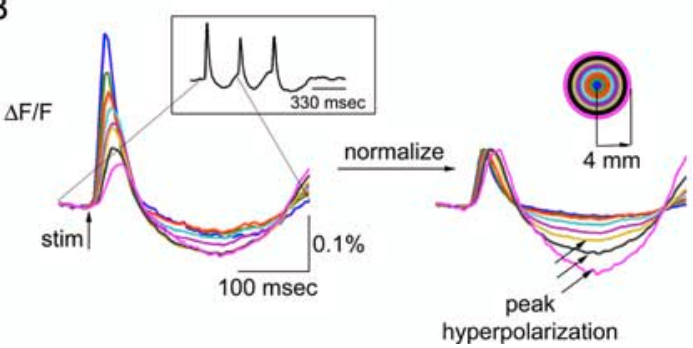

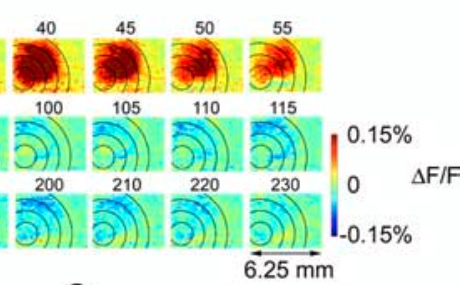

C

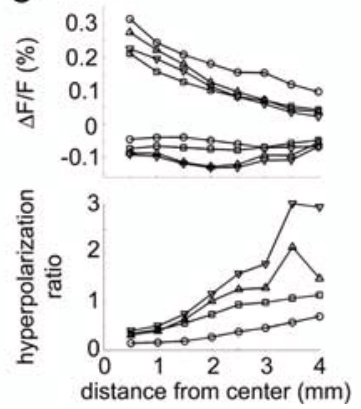

Figure 2. Voltage-sensitive dye measurements of neuronal activity. $\boldsymbol{A}$, Spatiotemporal evolution of VSD signal in response to the first of the three stimuli in a train. Time (in milliseconds) relative to stimulus onset $(t=0)$ is indicated above images. One hundred trials were averaged. The images were spatially smoothed using a Gaussian kernel of $120 \mu \mathrm{m}$ full width at $e^{-1}$ of peak amplitude. The color scale is expressed as fluorescence percentage change relative to the baseline $(\Delta F / F)$. An image of raw vasculature corresponding to functional frames is shown in the lower right corner. L, Lateral; M, medial; $R$, rostral. $\boldsymbol{B}$, Signal time courses extracted from $0.5 \mathrm{~mm}$ concentric rings around the center of the response. The center was estimated using the earliest response $(t=10 \mathrm{~ms})$. The signal time course in response to the first stimulus in a train of three is shown (see inset on the left). The rings are superimposed on images in $\boldsymbol{A}$. Every other ring is shown. In the right panel, the initial depolarization for each ring is normalized to $0.1 \%$. Black arrows label peak hyperpolarization. The inset on the right shows the color code. C, Peak depolarization and hyperpolarization (top) and the hyperpolarization ratio (bottom; see Results) as a function of distance from the center. Data from four animals are superimposed (circles, squares, upward triangles, and downward triangles).

larization ("hyperpolarization ratio") increased steadily in all four subjects up to $3.5 \mathrm{~mm}$ from the center. Comparison of the hyperpolarization ratio to the hemodynamic data (Fig. 1C) within the same range of distances $(0-4 \mathrm{~mm})$ reveals a correlation of negative hemodynamic signals (evoked decrease in blood oxygenation and volume) with relative hyperpolarization (relative neuronal inhibition).

\section{Laminar electrode array recordings}

To test whether the surround hyperpolarization observed with VSD imaging is accompanied by a decrease in firing rates, we performed laminar electrophysiological recordings in two animals after VSD imaging. MUA and LFP were recorded using two linear (depth) electrode arrays (Pettersen et al., 2006; Einevoll et al., 2007). The first array was inserted at the center of the active area. The second array was inserted $\sim 3 \mathrm{~mm}$ posterior relative to the first, in the area in which surround hyperpolarization was observed with VSD imaging. Each array had 23 electrodes separated by $100 \mu \mathrm{m}$ in depth. In agreement with previous studies in barrel cortex (Simons and Carvell, 1989; Webber and Stanley, 2004; Devor et al., 2005), we observed no change in MUA in the surround (Fig. 3A, top, supplemental Fig. 2, available at www.jneurosci.org as supplemental material). There was a small LFP response in the surround (Fig. $3 A$, bottom, supplemental Fig. 2, available at www.jneurosci.org as supplemental material). Given a large point spread function of LFP, this signal is likely to represent a "pick up" of the response in the center rather than local activity in the surround ((Pettersen et al., 2006) (for review, see Logothetis, 2002). It should be noted that VSD and LFP measure different aspects of neuronal activity in that VSD reflects mean membrane polarization, strongly weighted toward super- ficial cortical layers, whereas LFP are determined by the spatial distribution of transmembrane currents in a volume around the electrodes (Nicholson and Freeman, 1975; Pettersen et al., 2006; Einevoll et al., 2007).

\section{Two-photon microscopy}

We used two-photon laser-scanning microscopy to relate the center-surround hemodynamic and neuronal response pattern, as revealed with VSD imaging, to dilation changes on the level of single blood vessels. Before two-photon microscopy, we performed electrophysiological mapping using a surface ball electrode to determine the center of evoked neuronal activity (Fig. 4). Then, we measured diameter changes in single visually identified surface arterioles. Evoked diameter changes were measured at 216 locations (arterioles) in seven subjects. The baseline diameter of the measured arterioles ranged from 4 to $80 \mu \mathrm{m}$; however, most of the measurements $(86 \%)$ were performed on small arterioles with the baseline diameter $<30 \mu \mathrm{m}$. Figure 5 shows stimulusevoked temporal profiles of diameter change in arterioles located at different distances from the maximal neuronal response (evaluated by surface potential measurements using a ball electrode) in one subject. At virtually all locations, the initial dilation was followed by a subsequent constriction. The relative strength of dilation and constriction varied as a function of distance from the center of neuronal activity. The greatest dilation was observed at the location of the strongest neuronal response. At this location, large-amplitude dilation was followed by a small-amplitude constriction. The amplitude and the duration of the vasodilation decreased with an increase in distance from the center. The vasoconstriction, however, was in particular evident in remote locations relative to the center of neuronal activity. This is further illustrated in Figure $6 \mathrm{~A}$ for another subject. The figure shows an average of arteriolar diameter changes within three rings around the center of neuronal response. Normalization of the initial dilation amplitude revealed that relative to the initial dilation the subsequent constriction was greater in the surround regions (Fig. $6 A$, right, black arrows). Figure $6 B$ shows peak dilation (black dots) and peak constriction (red dots) at every measured location for all 216 measurements. Both dilation and constriction tended to decrease as a function of distance. However, as evident from the linear regression slope $(-2.8$ and 0.1 for dilation and constriction, respectively), the dilation decreased faster.

Superposition of the arteriolar responses from different locations shows that the onset and return to the baseline of the composite response were constant regardless of the degree of dilation and constriction (Fig. 6A). At the same time, the vasoconstriction reached peak faster further away from the center of neuronal activity (Fig. 6 A, supplemental Fig. 3, available at www.jneurosci. org as supplemental material). In fact, the response at the furthest measured locations ( $>3 \mathrm{~mm}$ from the center) was almost completely dominated by vasoconstriction. Theoretically, such behavior can be obtained assuming two antagonistic (competing) 
A
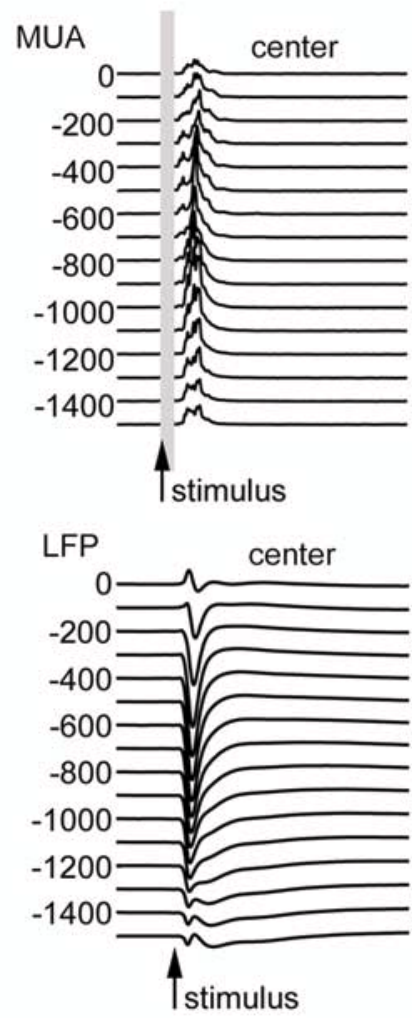

B
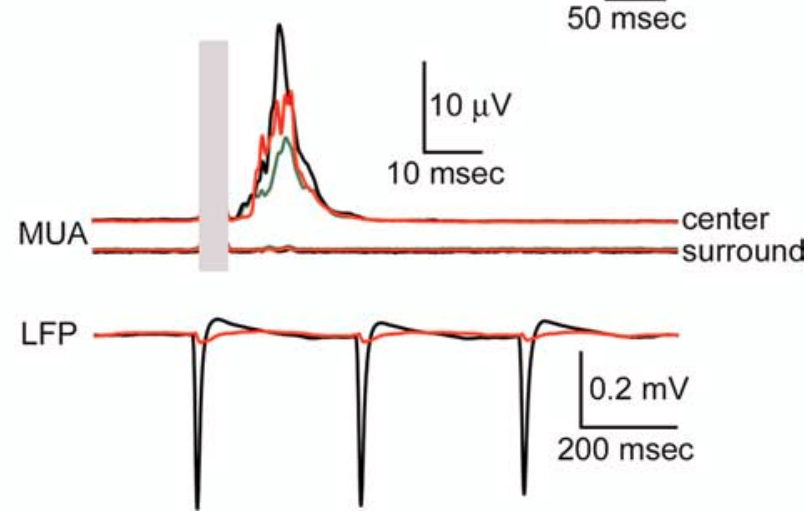

Figure 3. Laminar array recordings of MUA and LFP. A, Top, Averaged MUA response from the center (left) and the surround (right). Left and right columns are plotted on the same scale. The response to the first of the threestimuli in a train is shown. Each trace represents a recording from one single electrode in the array. Corresponding cortical depth is indicated on the left. The top electrode was positioned on the brain surface (depth, 0). Recordings down to $1500 \mu \mathrm{m}$ are shown. Fivehundred stimulus trials were averaged. The electrical stimulus artifact is covered by gray rectangles. Arrows denote stimulus onset. Bottom, Averaged LFP responsefrom the center (left) and the surround (right). Conventions are the same as for MUA. $\boldsymbol{B}$, Top, Enlarged MUA response averaged from supragranular (depth of $0-400 \mu \mathrm{m}$; green), granular (depth of $500-900 \mu \mathrm{m}$; black), and infragranular (depth of $900-1500 \mu \mathrm{m}$; red) layers. The top and bottom traces correspond to the recordings from the center and the surround, respectively. Bottom, Enlarged LFP response averaged from 200 to 500 $\mu \mathrm{m}$ (layer II/III). Responses from the center (black) and the surround (red) are superimposed. Responses to all three consecutive stimuli are shown.

and temporally overlapping forces, $D$ (for vasodilation) and $C$ (for vasoconstriction), with effective response time courses described as follows:

$$
D=\left(\frac{t-t_{0}}{\tau_{D}}\right)^{3} \times e^{-\left(t-t_{0}\right) / \tau_{D}}, C=-\left(\frac{t-t_{0}}{\tau_{C}}\right)^{3} \times e^{-\left(t-t_{0}\right) / \tau_{C}},
$$

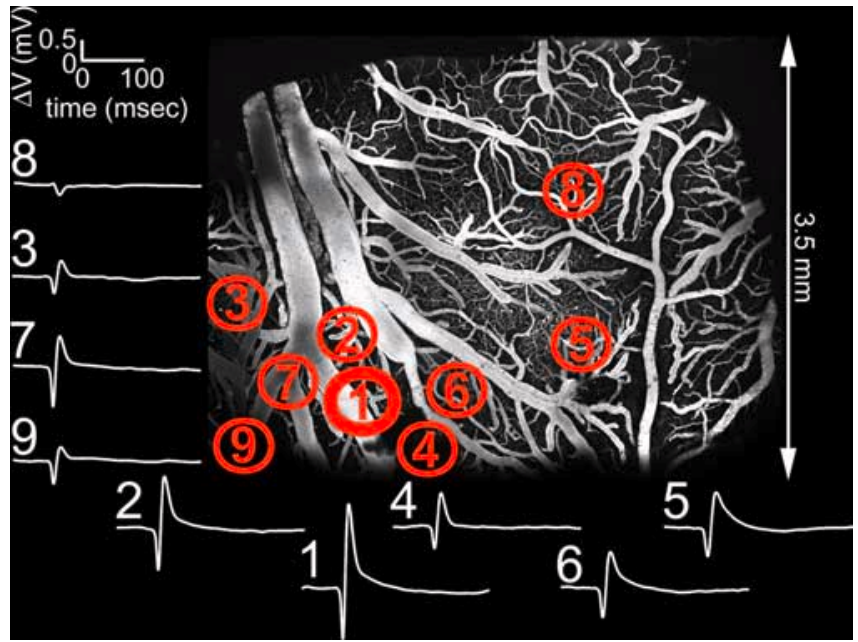

Figure 4. Mapping of the center of the evoked neuronal response using ball electrode measurements of surface potentials. Measurements (white traces) from nine different locations are shown. The traces are numbered in order they were recorded. The strongest amplitude and fastest rise time was observed at location 1, corresponding to the center. The locations are overlaid on the two-photon microscopy image of vasculature within the exposure. The image was calculated as a maximum intensity projection of an image stack of $0-300 \mu \mathrm{m}$ in depth. Individual images were acquired every $10 \mu \mathrm{m}$.

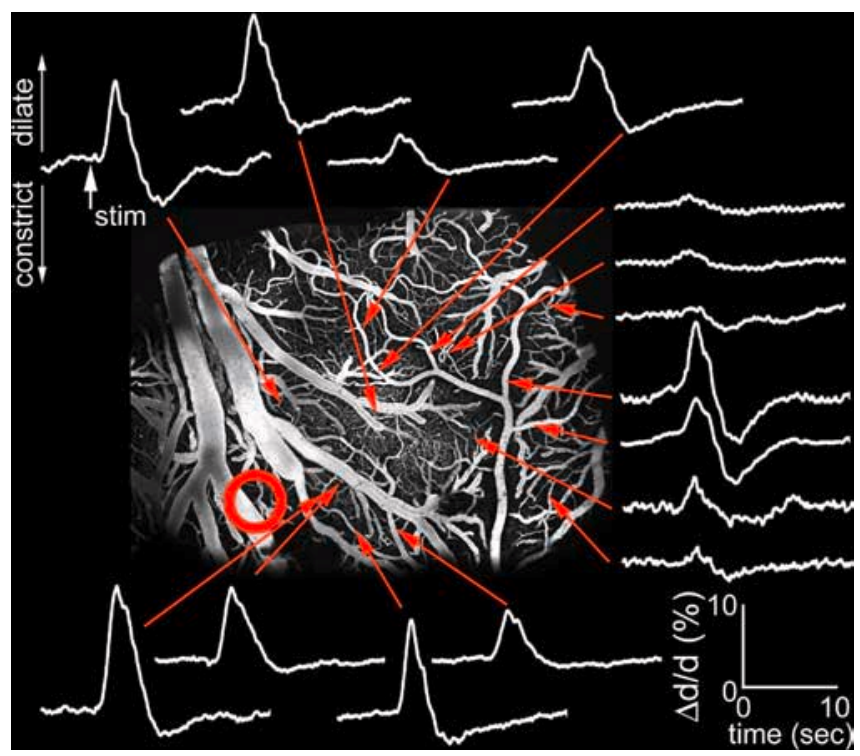

Figure 5. Arteriolar diameter change as a function of distance from the center of evoked neuronal response. White traces show percent diameter change relative to the baseline $(\Delta d / d)$ at different locations indicated by red arrows. Dilation and constriction are plotted up and down, respectively. The red circle shows the center of neuronal response. Ball electrode mapping of the center of the neuronal response for the same subject is shown in Figure 4.

where $t_{0}$ is the initial delay after stimulus onset, $t \geq t_{0}$, and $\tau_{D}$ and $\tau_{C}$ are dilation and constriction time constants, respectively.

Thus, the diameter change profiles

$$
\frac{\Delta d}{d}
$$

at each spatial location can be estimated by fitting vascular diameter data as a linear combination of $D$ and $C$ as follows:

$$
\frac{\Delta d}{d}=A \times D+B \times C,
$$


A

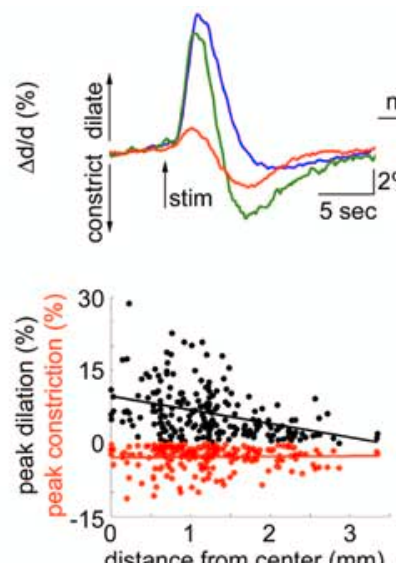

C

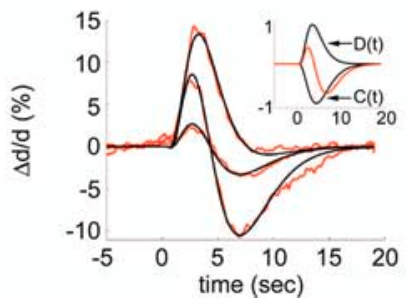

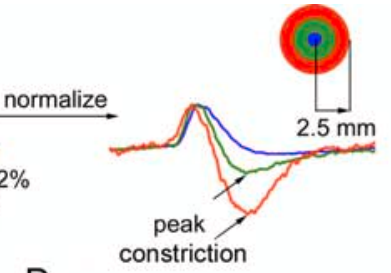

D
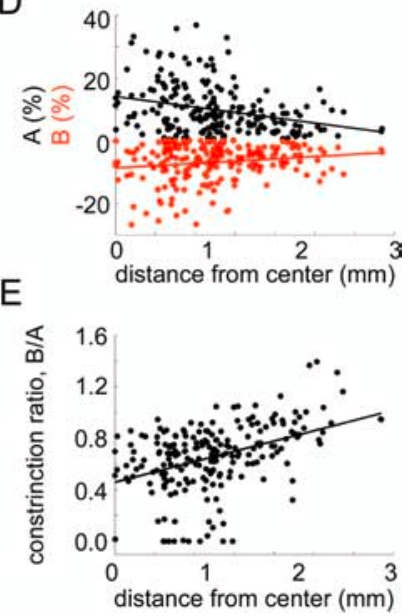

Figure 6. Arteriolar vasodilatation and vasoconstriction as a function of distance from the center of evoked neuronal response. $A$, An average of arteriolar diameter changes $(\Delta d / d)$ within $0.5 \mathrm{~mm}$ from the center of neuronal response (blue), in a $0.5-1.5 \mathrm{~mm}$ ring around the center (green), and 1.5-2.5 mm ring around the center (red) for one subject. In the right panel, the initial dilation for each ring is normalized to $3 \%$. The inset on the right shows the color code. Dilation and constriction are plotted up and down, respectively. $\boldsymbol{B}$, Peak dilation (top) and peak constriction (bottom) as a function of distance (in millimeters from the center of evoked neuronal response). Each dot represents a measurement from a single arteriole. Data from seven animals are superimposed. C, Three examples of the fit to the data. The fitting procedure is described in the text. The inset shows the functions C (positive) and D (negative) used to fit the data. The sum of the two normalized by the maximum of the sum of their absolute values is shown in red. $\boldsymbol{D}$, Linear coefficients $A$ (top) and $B$ (bottom) as a function of distance (in millimeters from the center of the evoked neuronal response). $B$ values are mirrored relative to the horizontal axis for better visualization (they are in fact positive). Data from seven animals are superimposed. $\boldsymbol{E}$, The ratio of constriction to dilatation estimated as $B / A$ as a function of distance. Each dot represents a measurement from a single arteriole. Data from seven animals are superimposed.

where $A$ and $B$ are non-negative weighting constants. Figure $6 C$ demonstrates the goodness of the fit using an example of three different profiles. The results of the fitting procedure described above explained $90 \%$ of the variance using all 216 temporal profiles. The best fit was obtained using $t_{0}=0.6 \mathrm{~s}, \tau_{D}=0.1 \mathrm{~s}$, and $\tau_{C}=1.4 \mathrm{~s}$.

Similarly to the measured peak dilation and peak constriction (Fig. 6B), the estimated values of $A$ and $B$ decreased as a function of distance from the center of the neuronal response $\left(p<1 e^{-5}\right.$ and $p<0.01$ for $A$ and $B$ respectively) (Fig. $6 D$ ). The estimated values were larger than measured peak dilation and constriction (Fig. $6 \mathrm{~B}$ ) because of the temporal overlap of antagonistic effects.

Comparison of single-arteriole diameter response profiles to the VSD imaging results reveals that both the hyperpolarization ratio (Fig. $2 C$ ) and the ratio of $B$ to $A$ ("constriction ratio") (Fig. $6 E$ ) increased with an increase in distance from the center within the range of the two-photon measurements (up to $3.5 \mathrm{~mm}$ from the center). Specifically, predominant vasoconstriction (constriction ratio $>1$ ) was observed $>2 \mathrm{~mm}$ away from the center, at locations that would correspond to the surround hyperpolarization (inhibition). Comparison of the constriction ratio (Fig. $6 E$ ) to the hemodynamic imaging data (Fig. 1C) within the same

range of distances $(0-3.5 \mathrm{~mm})$ reveals a correspondence of negative hemodynamic signals with a predominant vasoconstriction.

\section{Discussion}

Our results demonstrate that (1) increases in blood oxygenation and volume occur at locations with predominant neuronal excitation and arteriolar vasodilation, and (2) decreases in blood oxygenation and volume occur at locations with predominant neuronal inhibition and arteriolar vasoconstriction. Our stimulus evoked a surround net oxygenation decrease that would correspond to a negative BOLD fMRI signal (Fig. 1). This observation is in agreement with a previous study by Devor et al. (2005), albeit in a different sensory area using a different sensory stimulus. The neuronal response imaged using VSD also exhibited a centersurround structure with a central region of net neuronal depolarization surrounded by net hyperpolarization (Fig. 2). This finding is in agreement with a previous VSD imaging study in a different sensory area (Derdikman et al., 2003). As a means to determine a common denominator to these two effects, we measured diameter changes of single arterioles with two-photon microscopy. The measurements revealed that at every location within the exposure the arteriolar response was composed of a dilatory and constrictive phases. The relative strength of dilation and constriction varied as a function of distance from the center of neuronal activity. Although at the center the response was mostly dilatory, it was dominated by vasoconstriction at distant locations (Figs. 5, 6).

The antagonistic center-surround behavior, a good fit to the data assuming a combination of two antagonistic forces (Fig. $6 C-E$ ), and a covariance of the inhibition and constriction ratios as a function of distance from the center of evoked neuronal response suggest that evoked neuronal excitation is associated with arteriolar vasodilation, whereas evoked neuronal inhibition is associated with arteriolar vasoconstriction. We used a linear regression to illustrate trends in the behavior of dilation, constriction, and the constriction ratio as a function of distance. However, this does not imply a linear relationship.

Our focus on arterioles is motivated by the fact that their walls contain smooth muscle cells, a substrate for active diameter control by neurovascular mediators (for review, see Iadecola, 2004; Lauritzen, 2005; Hamel, 2006). Therefore, the observation that stimulus-evoked diameter change occurred on the arterial side suggests, but does not prove, the presence of active rather than passive processes. In the present study, we chose to examine surface vessels to facilitate the comparison with spectroscopic imaging that has high sensitivity to the cortical surface (Polimeni et al., 2005). The surface vessels have a lesser specificity to cortical columns and larger "upstream" dilation effects (Iadecola, 2004) than diving arterioles and their branches that feed well defined territories (Nishimura et al., 2007). Both of these factors are likely to contribute to the variability of our measurements.

Previous studies have demonstrated surround inhibition using VSD imaging (Takashima et al., 2001; Derdikman et al., 2003). Although hyperpolarization observed with VSD imaging is interpreted as neuronal inhibition (Grinvald et al., 1982), it serves as a low bound because the contribution of "shunting" inhibition does not cause appreciable hyperpolarization (Carandini and Heeger, 1994). In our study, we estimated inhibition using the response to the first stimulus pulse in a train. Although consecutive pulses elicited additional neuronal activity, spatial sharpening of the response has been shown previously after repeated stimulation (Kleinfeld and Delaney, 1996; Sheth et al., 
1998). Therefore, surround inhibition is not expected to decrease during a repetitive stimulus.

A body of evidence indicates that neurotransmitter and neuropeptide release during synaptic transmission, including a presynaptic and postsynaptic dendritic release, is a major factor in the initiation of the hemodynamic response (Cauli et al., 2004; Hamel, 2004, 2006; Iadecola, 2004). Thus, arteriolar dilation and constriction can be an end-point result of the release of vasoactive substances related to activity of certain populations (types) of neurons differentially engaged in network response in center and in surround cortical columns. The observation of the covariance of the inhibition and constriction ratios does not necessarily imply that excitatory and inhibitory neurons release dilatory and constrictive mediators, respectively. Although it has been shown that pyramidal cortical neurons express NMDA receptors and generate a vasodilator, NO (Iadecola, 2004), and that some of the inhibitory interneurons cause vasoconstriction after stimulation in vitro (Hamel, 2006), the role of different types of neurons in neurovascular communication needs additional in vivo investigation. Moreover, neurovascular signaling pathways involve non-neuronal cell types such as astrocytes (Wang et al., 2006) that might account for the well known $>0.5 \mathrm{~s}$ delay in the onset of the hemodynamic response and might play a critical role in the transformation of activity of neuronal circuits into vasodilation and vasoconstriction (Raichle and Mintun, 2006).

In agreement with the study by Devor et al. (2005) using tactile stimulation of a single whisker, the spectroscopic hemodynamic measures ( $\mathrm{Hb}, \mathrm{HbO}$, and $\mathrm{HbT}$ ) showed an antagonistic centersurround activation pattern in which the central area of the response was surrounded by an area of the opposite sign (Fig. 1). Evoked decrease in oxygenation, known as "negative BOLD" in fMRI literature (Shmuel et al., 2006), was observed at distances $>2 \mathrm{~mm}$ from the center. The area at the border between the positive center and the negative surround exhibited temporally biphasic signals. This is in contrast to our previous observations of a space-time separable center-surround structure in the primary vibrissa cortex. The difference can be attributed to differences in the somatosensory stimulus.

Comparison of the hemodynamic data (Fig. 1) to the hyperpolarization ratio (Fig. 2C) within the same range of distances $(0-4 \mathrm{~mm})$ reveals a correlation of negative hemodynamic signals with relative hyperpolarization (neuronal inhibition). Likewise, comparison of the hemodynamic data to the constriction ratio reveals a correlation of negative hemodynamic signals with a predominant vasoconstriction. In contrast to single-arteriole diameter change, the oxygenation and HbT change within the radius of $2 \mathrm{~mm}$ from the center did not exhibit a biphasic behavior, but always showed an increase. The lack of negative HbT can be explained by a compensating (passive) diameter change on the venous side and also by possible evoked changes in hematocrit (Hillman et al., 2007). Evoked change in oxygenation results not only from the arterial diameter change, but also from change in flow and the cerebral metabolic rate of oxygen consumption. Thus, quantitative interpretation of BOLD as diameters changes of single vessels requires a further effort including microscopic measurements of vascular diameter, blood flow velocity, and oxygenation in intravascular and extravascular space, ideally in the framework of realistic vascular trees.

Neuronal inhibition is not always accompanied by a decrease in measured population firing rates. Both a study by Devor et al. (2005) in the rat barrel cortex and the present data from the forepaw region of the SI (Fig. 3) demonstrate no decrease in MUA in hemodynamically negative areas. Because of the low baseline firing rate (Simons and Carvell, 1989), evoked inhibition in rat SI is not accompanied by MUA decrease. In contrast, in monkey visual cortex under conditions of elevated baseline, Shmuel et al. (2006) reported a decrease in MUA in areas with a negative BOLD signal change. The common denominator in these studies is the evoked neuronal inhibition, which may or may not be associated with decreased overall neuronal firing rates, as measured by MUA, depending on the baseline activity. Therefore, inhibitory synaptic activity, rather than a decrease in firing rate per se, appears to drive vasoconstriction and associated oxygenation decrease ("negative BOLD").

A number of factors in our study could affect the magnitude of the hemodynamic response in comparison with awake behaving animals in a natural environment. Among them are anesthesia conditions, intracranial pressure (caused by the draining of CSF from the fourth ventricle), unknown state of cholinergic modulation, and the use of an electrical forepaw stimulus. The present study used $\alpha$-chloralose, an agent of choice for hemodynamic imaging because of its lesser effects on cardiovascular, respiratory and reflex functions, and functional-metabolic coupling (Covert et al., 1992; Ueki et al., 1992; Lindauer et al., 1993). However, the spatially biphasic center-surround hemodynamic pattern of $\mathrm{HbO}, \mathrm{Hb}$, and $\mathrm{HbT}$ reported here is consistent with previous reports by Devor et al. $(2003,2005)$ from primary vibrissa cortex that used either $\alpha$-chloralose or urethane anesthesia and a more natural single-vibrissa stimulus. Therefore, the center-surround pattern is likely to be a preserved phenomenon across different somatosensory areas, anesthesia states, and stimulus types.

To conclude, our results suggest that positive and negative BOLD fMRI signals may be interpreted, respectively, as functional excitation and inhibition, that active vasoconstriction underlies the negative BOLD signal, and that the release of vasoactive compounds associated with evoked excitation and inhibition initiates the neurovascular signal transduction pathway(s) leading, respectively, to vasodilation and vasoconstriction. Additional in vivo studies using recent advancement in labeling and stimulation strategies of identified cell types are needed to address the molecular mediators in neurovascular cascade.

\section{References}

Attwell D, Iadecola C (2002) The neural basis of functional brain imaging signals. Trends Neurosci 25:621-625.

Brecht M, Sakmann B (2002) Dynamic representation of whisker deflection by synaptic potentials in spiny stellate and pyramidal cells in the barrels and septa of layer 4 rat somatosensory cortex. J Physiol (Lond) 543:49-70.

Brecht M, Roth A, Sakmann B (2003) Dynamic receptive fields of reconstructed pyramidal cells in layers 3 and 2 of rat somatosensory barrel cortex. J Physiol (Lond) 553:243-265.

Carandini M, Heeger DJ (1994) Summation and division by neurons in primate visual cortex. Science 264:1333-1336.

Cauli B, Tong XK, Rancillac A, Serluca N, Lambolez B, Rossier J, Hamel E (2004) Cortical GABA interneurons in neurovascular coupling: relays for subcortical vasoactive pathways. J Neurosci 24:8940-8949.

Chaigneau E, Oheim M, Audinat E, Charpak S (2003) Two-photon imaging of capillary blood flow in olfactory bulb glomeruli. Proc Natl Acad Sci USA 100:13081-13086.

Covert RF, Schreiber MD, Leff AR, White SR, Munoz NM, Torgerson LJ (1992) Oxygen metabolism and catecholamine secretion during chloralose anesthesia in lambs. J Dev Physiol 17:125-132.

Derdikman D, Hildesheim R, Ahissar E, Arieli A, Grinvald A (2003) Imaging spatiotemporal dynamics of surround inhibition in the barrels somatosensory cortex. J Neurosci 23:3100-3105.

Devor A, Dunn AK, Andermann ML, Ulbert I, Boas DA, Dale AM (2003) Coupling of total hemoglobin concentration, oxygenation, and neural activity in rat somatosensory cortex. Neuron 39:353-359.

Devor A, Ulbert I, Dunn AK, Narayanan SN, Jones SR, Andermann ML, Boas 
DA, Dale AM (2005) Coupling of the cortical hemodynamic response to cortical and thalamic neuronal activity. Proc Natl Acad Sci USA 102:3822-3827.

Dunn AK, Devor A, Bolay H, Andermann ML, Moskowitz MA, Dale AM, Boas DA (2003) Simultaneous imaging of total cerebral hemoglobin concentration, oxygenation, and blood flow during functional activation. Opt Lett 28:28-30.

Einevoll GT, Pettersen KH, Devor A, Ulbert I, Halgren E, Dale AM (2007) Laminar population analysis: estimating firing rates and evoked synaptic activity from multielectrode recordings in rat barrel cortex. J Neurophysiol 97:2174-2190.

Faraci FM, Breese KR (1993) Nitric oxide mediates vasodilatation in response to activation of $\mathrm{N}$-methyl-D-aspartate receptors in brain. Circ Res 72:476-480.

Faraci FM, Heistad DD (1998) Regulation of the cerebral circulation: role of endothelium and potassium channels. Physiol Rev 78:53-97.

Ferezou I, Bolea S, Petersen CC (2006) Visualizing the cortical representation of whisker touch: voltage-sensitive dye imaging in freely moving mice. Neuron 50:617-629.

Filosa JA, Bonev AD, Straub SV, Meredith AL, Wilkerson MK, Aldrich RW, Nelson MT (2006) Local potassium signaling couples neuronal activity to vasodilation in the brain. Nat Neurosci 9:1397-1403.

Grinvald A, Manker A, Segal M (1982) Visualization of the spread of electrical activity in rat hippocampal slices by voltage-sensitive optical probes. J Physiol (Lond) 333:269-291.

Hamel E (2004) Cholinergic modulation of the cortical microvascular bed. Prog Brain Res 145:171-178.

Hamel E (2006) Perivascular nerves and the regulation of cerebrovascular tone. J Appl Physiol 100:1059-1064.

Harel N, Lee SP, Nagaoka T, Kim DS, Kim SG (2002) Origin of negative blood oxygenation level-dependent fMRI signals. J Cereb Blood Flow Metab 22:908-917.

Hillman EM, Devor A, Bouchard MB, Dunn AK, Krauss GW, Skoch J, Bacskai BJ, Dale AM, Boas DA (2007) Depth-resolved optical imaging and microscopy of vascular compartment dynamics during somatosensory stimulation. NeuroImage 35:89-104.

Iadecola C (2004) Neurovascular regulation in the normal brain and in Alzheimer's disease. Nat Rev Neurosci 5:347-360.

Kleinfeld D, Delaney KR (1996) Distributed representation of vibrissa movement in the upper layers of somatosensory cortex revealed with voltage-sensitive dyes. J Comp Neurol 375:89-108.

Kleinfeld D, Mitra PP, Helmchen F, Denk W (1998) Fluctuations and stimulus-induced changes in blood flow observed in individual capillaries in layers 2 through 4 of rat neocortex. Proc Natl Acad Sci USA 95:15741-15746.

Lauritzen M (2005) Opinion: reading vascular changes in brain imaging: is dendritic calcium the key? Nat Rev Neurosci 6:77-85.

Lindauer U, Villringer A, Dirnagl U (1993) Characterization of CBF response to somatosensory stimulation: model and influence of anesthetics. Am J Physiol 264:H1223-H1228.

Logothetis NK (2002) The neural basis of the blood-oxygen-leveldependent functional magnetic resonance imaging signal. Philos Trans $\mathrm{R}$ Soc Lond B Biol Sci 357:1003-1037.

Manns ID, Sakmann B, Brecht M (2004) Sub- and suprathreshold receptive field properties of pyramidal neurones in layers $5 \mathrm{~A}$ and $5 \mathrm{~B}$ of rat somatosensory barrel cortex. J Physiol (Lond) 556:601-622.

Moore CI, Nelson SB (1998) Spatio-temporal subthreshold receptive fields in the vibrissa representation of rat primary somatosensory cortex. J Neurophysiol 80:2882-2892.

Mulligan SJ, MacVicar BA (2004) Calcium transients in astrocyte endfeet cause cerebrovascular constrictions. Nature 431:195-199.

Nicholson C, Freeman JA (1975) Theory of current source-density analysis and determination of conductivity tensor for anuran cerebellum. J Neurophysiol 38:356-368.

Nishimura N, Schaffer CB, Friedman B, Tsai PS, Lyden PD, Kleinfeld D (2006) Targeted insult to subsurface cortical blood vessels using ultrashort laser pulses: three models of stroke. Nat Methods 3:99-108.
Nishimura N, Schaffer CB, Friedman B, Lyden PD, Kleinfeld D (2007) Penetrating arterioles are a bottleneck in the perfusion of neocortex. Proc Natl Acad Sci USA 104:365-370.

Peppiatt CM, Howarth C, Mobbs P, Attwell D (2006) Bidirectional control of CNS capillary diameter by pericytes. Nature 443:700-704.

Petersen CC, Grinvald A, Sakmann B (2003) Spatiotemporal dynamics of sensory responses in layer $2 / 3$ of rat barrel cortex measured in vivo by voltage-sensitive dye imaging combined with whole-cell voltage recordings and neuron reconstructions. J Neurosci 23:1298-1309.

Pettersen KH, Devor A, Ulbert I, Dale AM, Einevoll GT (2006) Currentsource density estimation based on inversion of electrostatic forward solution: effects of finite extent of neuronal activity and conductivity discontinuities. J Neurosci Methods 154:116-133.

Polimeni JR, Granquist-Fraser D, Wood RJ, Schwartz EL (2005) Physical limits to spatial resolution of optical recording: clarifying the spatial structure of cortical hypercolumns. Proc Natl Acad Sci USA 102:4158-4163.

Raichle ME, Mintun MA (2006) Brain work and brain imaging. Annu Rev Neurosci 29:449-476.

Rancillac A, Rossier J, Guille M, Tong XK, Geoffroy H, Amatore C, Arbault S, Hamel E, Cauli B (2006) Glutamatergic control of microvascular tone by distinct GABA neurons in the cerebellum. J Neurosci 26:6997-7006.

Roy CS, Sherrington CS (1890) On the regulation of the blood-supply of the brain. J Physiol (Lond) 11:85-158.

Schaffer CB, Friedman B, Nishimura N, Schroeder LF, Tsai PS, Ebner FF, Lyden PD, Kleinfeld D (2006) Two-photon imaging of cortical surface microvessels reveals a robust redistribution in blood flow after vascular occlusion. PLoS Biol 4:e22.

Sheth BR, Moore CI, Sur M (1998) Temporal modulation of spatial borders in rat barrel cortex. J Neurophysiol 79:464-470.

Shmuel A, Yacoub E, Pfeuffer J, Van de Moortele PF, Adriany G, Hu X, Ugurbil K (2002) Sustained negative BOLD, blood flow and oxygen consumption response and its coupling to the positive response in the human brain. Neuron 36:1195-1210.

Shmuel A, Augath M, Oeltermann A, Logothetis NK (2006) Negative functional MRI response correlates with decreases in neuronal activity in monkey visual area V1. Nat Neurosci 9:569-577.

Simard M, Arcuino G, Takano T, Liu QS, Nedergaard M (2003) Signaling at the gliovascular interface. J Neurosci 23:9254-9262.

Simons DJ, Carvell GE (1989) Thalamocortical response transformation in the rat vibrissa/barrel system. J Neurophysiol 61:311-330.

Takano T, Tian GF, Peng W, Lou N, Libionka W, Han X, Nedergaard M (2006) Astrocyte-mediated control of cerebral blood flow. Nat Neurosci 9:260-267.

Takashima I, Kajiwara R, Iijima T (2001) Voltage-sensitive dye versus intrinsic signal optical imaging: comparison of optically determined functional maps from rat barrel cortex. NeuroReport 12:2889-2894.

Tsai PS, Nishimura N, Yoder EJ, Dolnick E, White A, Kleinfeld D (2002) Principles, design and construction of a two-photon laser-scanning microscope for in vitro and in vivo brain imaging. In: In vivo optical imaging of brain function (Frostig RD, ed), pp 113-171. Boca Raton, FL: CRC.

Ueki M, Mies G, Hossmann KA (1992) Effect of alpha-chloralose, halothane, pentobarbital and nitrous oxide anesthesia on metabolic coupling in somatosensory cortex of rat. Acta Anaesthesiol Scand 36:318-322.

Ulbert I, Halgren E, Heit G, Karmos G (2001) Multiple microelectroderecording system for human intracortical applications. J Neurosci Methods 106:69-79.

Wang X, Lou N, Xu Q, Tian GF, Peng WG, Han X, Kang J, Takano T, Nedergaard M (2006) Astrocytic $\mathrm{Ca}(2+)$ signaling evoked by sensory stimulation in vivo. Nat Neurosci 9:816-823.

Webber RM, Stanley GB (2004) Nonlinear encoding of tactile patterns in the barrel cortex. J Neurophysiol 91:2010-2022.

Zonta M, Angulo MC, Gobbo S, Rosengarten B, Hossmann KA, Pozzan T, Carmignoto G (2003) Neuron-to-astrocyte signaling is central to the dynamic control of brain microcirculation. Nat Neurosci 6:43-50. 